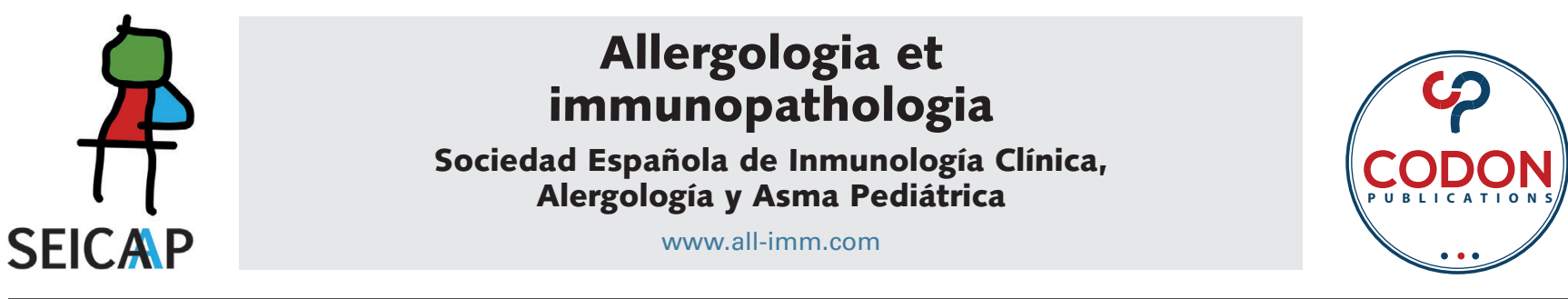

\title{
Taxifolin ameliorates sepsis-induced lung capillary leak through inhibiting the JAK/STAT3 pathway
}

\author{
Mengwen Shen ${ }^{\mathrm{a}}$, Baibai Lin ${ }^{\mathrm{b}}$, Fenghua Qian ${ }^{\mathrm{a}}$, Lei Zhao ${ }^{\mathrm{a}}$, Yao Xia, Yiming Qian ${ }^{\mathrm{a}}$
}

a Department of Emergency Medical, Yueyang Hospital of Integrated Traditional Chinese and Western Medicine Affiliated to Shanghai University of Traditional Chinese Medicine, Shanghai, China

${ }^{b}$ Department of Emergency Medical, Shanghai Baoshan District Hospital of Integrated Traditional Chinese and Western Medicine, Shanghai, China

Received 26 November 2021; Accepted 28 December 2021

Available online 1 March 2022

\section{KEYWORDS}

taxifolin;

lung capillary leak;

sepsis;

Th17/Treg balance;

JAK/STAT3 signaling

pathway

\begin{abstract}
Background: As a systemic inflammatory reaction, sepsis is associated with various organ dysfunctions. The capillary leakage and the imbalance between $\mathrm{T}$ helper 17 and regulatory T (Th17/Treg) cells are associated with sepsis-induced lung injury. Taxifolin (TXL) has been found to play a vital role in regulating this diverse disease. However, the detailed functioning and mechanism of TXL in regulating sepsis-induced lung capillary leak remain elusive.

Methods: Balb/c mice were used to establish sepsis-induced lung injury model through administration of lipopolysaccharide (LPS). The structure of lung tissues was observed by using hematoxylin \& eosin staining. Protein level and total cells in bronchoalveolar lavage fluid (BALF) were measured by bicinchoninic acid (BCA) protein assay kit and hematimetry assay, respectively. Quantitative real-time reverse transcription polymerase chain reaction and enzyme-linked immunosorbent assay were employed to detect the level of inflammatory cytokines. The content of Th17 and Treg cells were measured by flow cytometry analysis. Western blot assay was used to determine the protein level of retinoid-related orphan receptor-yt (RORYt), Forkhead box P3 (Foxp3), Janus kinase 2 (JAK2), phospho(p)-JAK2, signal transducer and activator of transcription 3 (STAT3), and phospho(p)-STAT3.

Results: Taxifolin effectively prolonged the survival period of sepsis mice and alleviated LPS-induced lung injury in a dose-dependent manner. Moreover, TXL reduced LPS-induced increase in protein levels and T cell content in BALF, and effectively restored the wet:dry ratio of lung tissue and tissue permeability. Treating with TXL notably inhibited the production of pro-inflammatory cytokines induced by sepsis and influenced the balance between Th17 and Treg cells. Furthermore, TXL treatment suppressed the activation of JAK/STAT3 signaling pathway in a dose-dependent manner.

Conclusion: Our findings revealed that TXL alleviated sepsis-induced capillary leak in the lungs of mice by regulating JAK/STAT3 signaling pathway.

(C) 2022 Codon Publications. Published by Codon Publications.
\end{abstract}

*Corresponding author: Yiming Qian, Department of Emergency Medical, Yueyang Hospital of Integrated Traditional Chinese and Western Medicine Affiliated to Shanghai University of Traditional Chinese Medicine, No. 110 Ganhe Road, Hongkou District, Shanghai 200437. China. Email address: qianyiming88888@163.com

https://doi.org/10.15586/aei.v50i2.550

Copyright: Shen $M$, et al.

License: This open access article is licensed under Creative Commons Attribution 4.0 International (CC BY 4.0). http://creativecommons.org/ 


\section{Introduction}

Sepsis, the leading global health issue, can lead to multiple organ dysfunctions, and is caused by dysregulated host response to infection., ${ }^{1,2}$ The death rate of patients because of sepsis, being approximately $40 \%$, is still very high, and its therapy primarily depends on supportive care. However, targeted therapies are required urgently to cut off the abnormal contact between host and pathogen for treating sepsis patients.

The loss of endothelial barrier is closely related to systemic inflammation, the primary cause of sepsis. ${ }^{3}$ Increased vascular permeability contributes to sepsis capillary leak syndrome (Clarkson Syndrome or SCLS), which has been demonstrated to be correlated with patient's prognosis. ${ }^{4}$ Additionally, capillary permeability is identified as a strictly regulated characteristic of microenvironment, which obviously changes with the progression of sepsis, thereby leading to blood outflow from vascular space to tissues.

During the progression of sepsis, various inflammatory cytokines are capable of disturbing the immune response and injuring multiple tissues and organs., ${ }^{5,6}$ Therefore, immune homeostasis undoubtedly plays an important role in the occurrence and development of SCLS. Previous studies have proved that $\mathrm{T}$ lymphocytes may be closely involved in immune dysregulation occurring in the early and late stages of sepsis.(7-10) For example, the balance between T helper 17 (Th17) and regulatory T (Treg) lymphocytes plays a vital role in the progress of sepsis. ${ }^{11,12}$ Mature Th17 cells could secret interleukin (IL)-17 and IL-6 to induce pro-inflammatory response, while Treg cells produce transforming growth factor-beta (TGF-B) and IL-10 to resist inflammation. ${ }^{9,12}$ This imbalance between Thl7 and Treg cells results in diverse inflammatory diseases.

Recent studies have revealed that traditional Chinese medicine (TCM) plays an important role in treating sepsis and regulating immune system. For instance, $\mathrm{Ni}$ et al. discovered that toddalolactone inhibited lipopolysaccharide (LPS)-induced sepsis and inflammatory response by inhibiting heterotrimeric G-protein beta subunit (HGB1)-nuclear factor kappa $B$ (NF-kB) signaling pathway. ${ }^{13}$ Besides, it has been reported that polygonumorientale $L$. (polygonaceae) can be considered as a useful TCM. ${ }^{14}$ Taxifolin (TXL), one of the active components extracted from the mature fruit of polygonaceae, exerts crucial biological activity. ${ }^{14}$ For example, TXL effectively alleviated iron overload-induced liver cell injury by decreasing inflammatory cytokines and enhancing liver cell regeneration. ${ }^{15}$ In addition, TXL ameliorates cisplatin-induced lung injury and reduces excessive oxidative stress in the lungs. ${ }^{16}$ However, the mechanism of TXL regulating SCLS is still unclear. It was first discovered that TXL remarkably suppressed sepsis-induced lung injury and inflammatory response.

Previous reports have revealed that TXL is able to inhibit the Janus kinase 2/signal transducer and activator of transcription 3 (JAK/STAT3) signaling pathway, and reduces the expression of retinoic acid receptor-related orphan nuclear receptor gamma (RORY), thereby decreasing the load of Th17 inflammatory phenotype in a sepsis model. ${ }^{17}$ Therefore, we hypothesized that TXL was capable of regulating the imbalance between Th17 and Treg cells through inhibiting the JAK/STAT3 axis. Interestingly, this investigation for the first time confirmed that $T X L$ altered the imbalance between Th17 and Treg cells by inhibiting JAK/STAT3 signaling pathway and alleviated the sepsis-induced pulmonary capillary leakage.

\section{Materials and Methods}

\section{Animals}

Male Balb/c mice, 8-10-week old, weighing 20-25 g, were brought from the Vital River (Beijing, China). All mice were cultured in rooms with a temperature of $20 \pm 2^{\circ} \mathrm{C}$ and 12-h day-night cycle and had free access to water and food for no less than 7 days to acclimate in the environment. All protocols were in accordance with the National Guidelines for the Use and Care of Laboratory Animals and approved by the Animal Ethics Committee of Yueyang Hospital of Integrated Traditional Chinese and Western Medicine Affiliated to Shanghai University of Traditional Chinese Medicine (Approval No.: YYLAC-2020-086).

\section{LPS-induced sepsis model}

In all, 25 male Balb/c mice were randomly divided into the following five groups (five mice per group): sham, LPS, LSP+2.5 mg/kg TXL (78666-25MG-F, Merck, Germany), LPS $+5 \mathrm{mg} / \mathrm{kg}$ TXL, and LPS+10 mg/kg TXL. LPS (30 mg/kg/ mouse; L23352; Invitrogen, USA) or TXL at particular concentration dissolved in $0.1 \mathrm{~mL}$ of sterile saline was intraperitoneally injected into indicated mice. Mice in the sham group were injected with equal volume of sterile saline. At $24 \mathrm{~h}$ post-sterile saline or LPS with or without TXL injection, mice were anesthetized with $50-\mathrm{mg} / \mathrm{kg}$ sodium pentobarbital and sacrificed with cervical dislocation method for the following assays. The flow chart of model establishment has been provided in Figure 1.

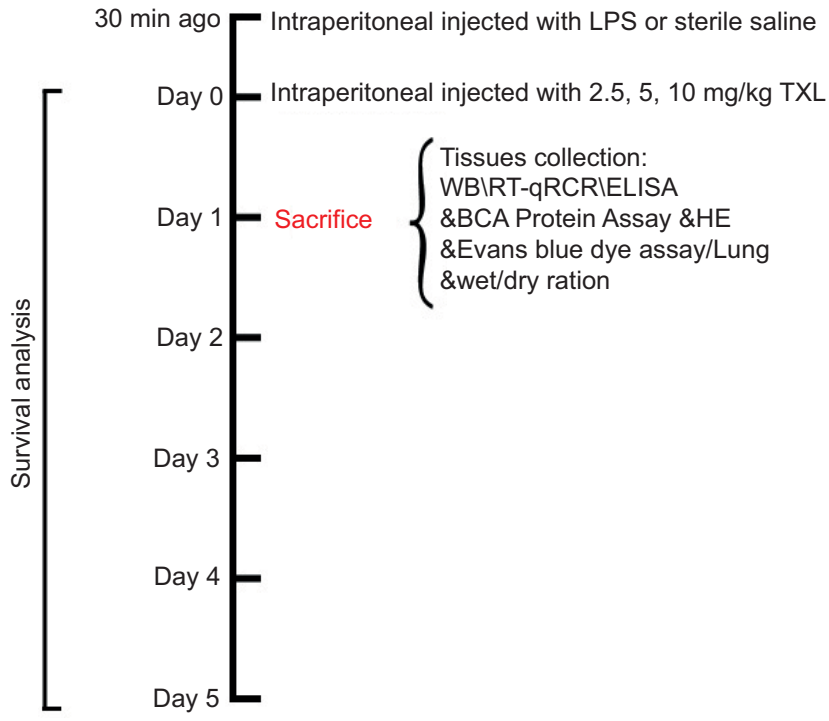

Figure 1 The flow chart of experimental method for establishing LPS-induced sepsis model. 


\section{Survival analysis}

Lipopolysaccharide-induced sepsis model was established in male mice according to the above-mentioned method. The survival rate of mice was recorded every 2 days for 6 days after the surgery. Finally, the survival curve was drawn according to survival rate.

\section{Hematoxylin \& eosin (H\&E) staining}

The protocol of H\&E staining was in accordance with the study conducted by Parikh et al. ${ }^{18}$ Briefly, lung tissues isolated from mice were fixed in $10 \%$ buffered formalin. After $24 \mathrm{~h}$, the tissues were dehydrated with graded ethanol and embedded into paraffin. Next, the paraffin was cut into $5-\mu \mathrm{m}$ slices and placed on slides. H\&E reagent was applied to stain lung tissue sections and observed under light microscope (JS-750T; LIOO, Germany).

\section{Bronchoalveolar lavage fluid (BALF) analysis}

Bronchoalveolar lavage fluid was isolated from each mouse $24 \mathrm{~h}$ after the mentioned injection. Male mice were sacrificed and the right lung of each mouse was ligated, and the left lung was rendered thrice into $0.5-\mathrm{mL}$ phosphate buffer saline (PBS) solution. Finally, the BALF was collected in a slow manner.

The protein level in BALF was measured by bicinchoninic acid (BCA) protein assay kit (23225; Thermo Scientific, USA) according to manufacturer's instructions.

For total cell count, the BALF was centrifuged at $600 \mathrm{~g}$ for $5 \mathrm{~min}$ and the cells were suspended in $0.5-\mathrm{mL}$ red blood cell lysis buffer. Next, the cells were again centrifuged as described above and suspended in $0.5-\mathrm{mL}$ PBS solution. Finally, cells were counted using Countess ${ }^{\mathrm{TM}} 3 \mathrm{FL}$ Automatic Cell Counter (AMQAF2000; Invitrogen).

\section{Evans blue dye assay}

Evans blue dye assay was applied to determine pulmonary permeability index; 20-mg/kg Evans blue dye (E2129-10G; Sigma, Germany) was injected intravenously into male mice for $30 \mathrm{~min}$ before anesthesia. Then the dye was collected from the lung lobes and incubated with $3-\mathrm{mL} / 100 \mathrm{~kg}$ formamide (17899; Thermo Scientific) for $24 \mathrm{~h}$ at room temperature. A spectrophotometer was used to detect the optical density of supernatant at a wavelength of $620 \mathrm{~nm}$, and Evans blue staining rate was estimated by comparing with standard absorbance value.

\section{Lung wet:dry ratio}

The lung wet:dry ratio was measured according to the study conducted by Thimmulappa et al. ${ }^{19}$ Briefly, the right lung was isolated and weighed for its wet weight. Then the right lung was dried at $60^{\circ} \mathrm{C}$ overnight and weighed for its dry weight. Finally, the wet:dry ratio was calculated by the following formula: wet weight/dry weight.
Quantitative real-time reverse transcription polymerase chain reaction ( $q R T-P C R)$

Total RNAs in BALF were collected by using TRIzol reagent (T9424-25ML; Sigma), and $2-\mu$ g total RNA sample was applied to reverse-transcribed into complementary DNA (CDNAs) with RT-PCR first strand cDNA synthesis kit (11483188001; Roche, Switzerland) according to manufacturer's protocol. qRT-PCR was conducted by incubating cDNAs with FastStart SYBR Green premix (4673484001; Roche). The mRNA level of specific genes was determined

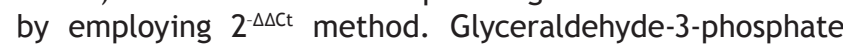
dehydrogenase (GAPDH) level was regarded as normalization. The primer sequences are listed in Table 1.

\section{Enzyme-linked immunosorbent assay (ELISA)}

Protein level of tumor necrosis factor alpha (TNF-a, 88-7324-88; Invitrogen), interleukin (IL)-6 (BMS213-2; Invitrogen), and IL-1B (RAB0308-1KT; Sigma) in BALF were measured by using specific ELISA kit as described by Zhang and Niu. ${ }^{20}$

\section{Flow cytometry analysis}

Bronchoalveolar lavage fluid extracted from mice was digested with enzymes, and undigested sections were filtered using strainers. Then, Mouse Th17/Treg phenotyping kit (560767; BD Biosciences, USA) was employed to stain Th17 and Treg cells according to the manufacturer's instructions. A flow cytometer (CytoFLEX; Beckman, USA) was used to record the number of specific cells $\left(1 \times 10^{4}\right.$ cells were tested).

\section{Western blot analysis}

Bronchoalveolar lavage fluid isolated from male mice was lysed using $1 \times$ radioimmunoprecipitation (RIPA) lysis buffer (20-188; Millipore, USA). Then the mixture was centrifuged at $4^{\circ} \mathrm{C}(12,000 \mathrm{~g}$ for $10 \mathrm{~min})$ and liquid supernatant was transferred into a separate centrifugal tube. The concentration

Table 1 The sequence of qRT-PCR primers.

\begin{tabular}{ll}
\hline Genes & Primers \\
\hline TNF- $a$ & F-5'-GGTGCCTATGTCTCAGCCTCTT-3' \\
& R-5'- GCCATAGAACTGATGAGAGGGAG-3' \\
IL-6 & F-5'- TACCACTTCACAAGTCGGAGGC-3 \\
& R-5'-CTGCAAGTGCATCATCGTTGTTC-3' \\
$I L-1 B$ & F-5'-TGGACCTTCCAGGATGAGGACA-3' \\
& R-5'-GTTCATCTCGGAGCCTGTAGTG-3 \\
$I L-17$ & F-5'-CAGACTACCTCAACCGTTCCAC-3' \\
& R-5'-TCCAGCTTTCCCTCCGCATTGA-3' \\
$I L-10$ & F-5'-GCTCTTACTGACTGGCATGAG-3' \\
& R-5'-CGCAGCTCTAGGAGCATGTG \\
\hline
\end{tabular}

F: forward, R: reverse. 
of total protein was estimated by using BCA protein assay kit (23225; Thermo Scientific). Protein sample, $20 \mu \mathrm{g}$, was separated by sodium dodecyl sulfate-polyacrylamide gel (SDS-PAGE) and transferred onto polyvinylidene fluoride (PVDF) membrane (3010040001; Roche). Next, 3\% non-fat milk dissolved in tris-buffered saline with $0.1 \%$ tween-20 (TBS-T) was introduced to block the membrane for $1 \mathrm{~h}$ at room temperature. Then the membrane was incubated with primary antibodies at $4^{\circ} \mathrm{C}$ overnight against retinoid-related orphan receptor-yt (RORyt, 1:1000, 562894; BD Biosciences), Forkhead box P3 (Foxp3, 1:1000, 14-5773; Invitrogen Antibodies), phospho(p)-JAK2 (1:1000, SAB4300124; MilliporeSigma, Germany), JAK (1:1000, 3230; Cell Signaling Technology, USA), phospho(p)-STAT3 (1:1000, 9145; Cell Signaling Technology), STAT3 (1:1000, 9139; Cell Signaling Technology), and B-actin (1:1000, 4970; Cell Signaling Technology). After being washed thrice with TBS-T, the membrane was incubated with anti-rabbit immunoglobulin $\mathrm{G}$ (lgG), horseradish peroxidase (HRP)-linked antibody (1:2000, 7074; Cell Signaling Technology) for $2 \mathrm{~h}$ at room temperature. Finally, the specific protein band was detected using Immobilon ECL ultra western HRP substrate (WBULS0100; Millipore). B-actin was regarded as the control protein, and the Gel pro 3.0 software was used to quantify protein level.

\section{Statistical analysis}

Statistical analysis was conducted with the Graphpad Prism 8.0 software. Statistical significance among multiple groups was evaluated using One-Way Analysis of Variance (ANOVA) followed by Tukey's post-hoc test, while statistical significance between two groups was analyzed by unpaired Student's $t$-test. All experiments were repeated for more than three times. $\mathrm{P}<0.05$ was considered as statistically significant.

\section{Results}

\section{TXL improved the survival time and lung injury of sepsis mice}

In order to confirm the detailed effect of TXL on the survival time of mice, we recorded survival of mice administrated with LPS $(30 \mathrm{mg} / \mathrm{kg})$ with or without indicated concentration of TXL treatment $(2.5,5$, or $10 \mathrm{mg} / \mathrm{kg})$ consecutively for 6 days. As shown in Figure 2A, 20\% of mice with LPS-induced sepsis survived for 6 days. TXL treatment $(2.5,5$, and $10 \mathrm{mg} / \mathrm{kg})$ remarkably elongated the 6-day survival rate of mice in a dose-dependent manner to $40 \%$, $60 \%$, and $80 \%$, respectively $(P<0.05, P<0.05)$.

Next, H\&E staining was employed to illustrate that LPS pretreatment badly damaged lung tissues, thickened the alveolar septum, and elicited inflammatory cytokine storm and disappearance of normal lung tissue structure and distinct hemorrhage in addition to pulmonary interstitial edema. Nevertheless, treatment with TXL significantly alleviated the LPS-induced severe lung injury of mice in a dose-dependent manner (Figure 2B). Collectively, our data demonstrated that TXL notably protected mice from lung injury caused by sepsis.

(A)

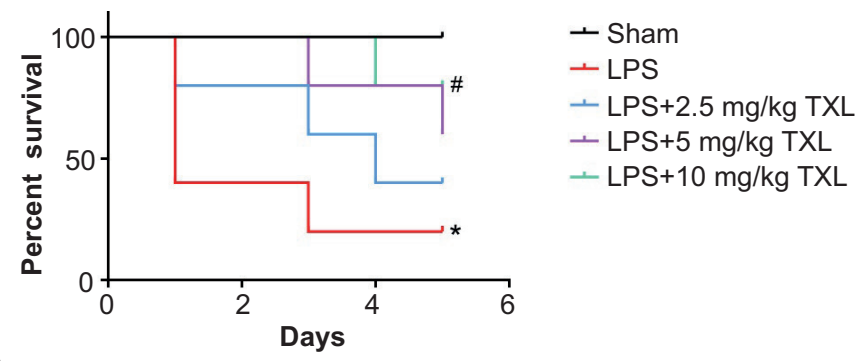

(B)

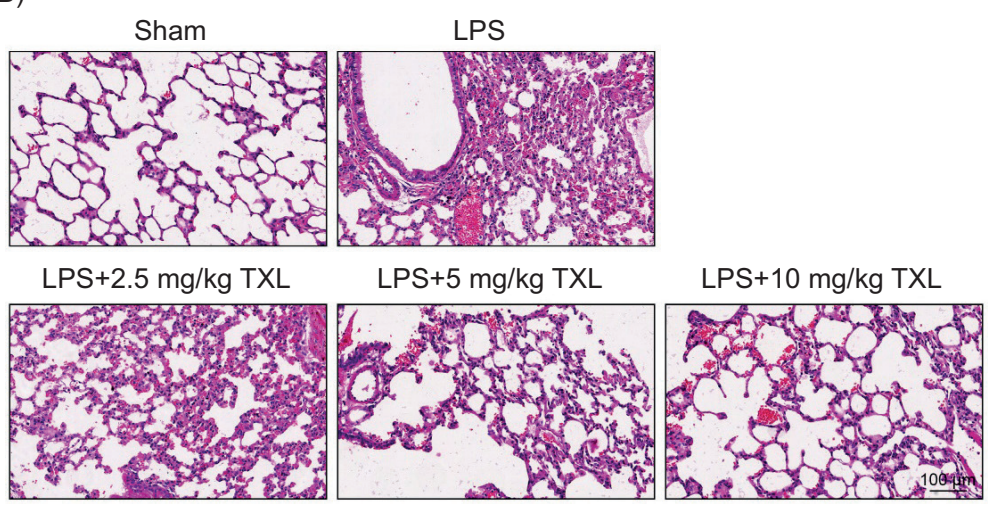

Figure 2 TXL improved survival rate and lung injury of mice in a model of sepsis. (A) The survival rate of mice in sepsis-induced lung injury model (LPS vs. sham "P $<0.05$, LPS $+10-\mathrm{mg} / \mathrm{kg}$ TXL vs. sham ${ }^{\#} P<0.05$ ). (B) The structure of lung tissue detected by H\&E staining. 


\section{$T X L$ relieved sepsis-induced capillary leakage in the lungs}

We subsequently detected the effect of TXL on capillary leakage in the lungs. Protein load in the BALF of mice was measured to reflect capillary permeability. The data demonstrated that LPS-induced sepsis injured lung capillaries, thereby increasing the content of protein in BALF, which was dramatically decreased by TXL treatment in a concentration-dependent manner $(P<0.01$, Figure $3 A)$. Then the total cells in BALF were estimated to evaluate inflammatory response, and the results revealed that LPSinduced sepsis increased the load of cells: $2.5-\mathrm{mg} / \mathrm{kg} \mathrm{TXL}$ did not affect cell count increased by sepsis, while 5 - and $10-\mathrm{mg} / \mathrm{kg}$ TXL obviously decreased the content of total cells in BLAF $(P<0.01$, Figure $3 B)$. Moreover, the lung wet:dry ratio was measured to assess the degree of pulmonary edema. The results suggested that LPS-induced sepsis increased wet:dry ratio, which was decreased by TXL treatment, especially high-dose TXL ( $P<0.01$, Figure $3 C)$. Finally, Evans blue staining was carried to evaluate changes in the permeability of lung tissues. Compared with the sham group, LPS increased Evans blue value whereas the value decreased in TXL-treated mice in a dose-dependent manner $(P<0.01, P<0.05$, Figure $3 D)$. The data implied that TXL relieved sepsis-induced lung capillary leakage.

\section{$T X L$ inhibited inflammatory response in BALF induced by sepsis}

The qRT-PCR assay was conducted to measure the level of inflammatory cytokines to determine the role of TXL in regulating inflammatory response. The data suggested that the level of pro-inflammatory cytokines (TNFa, IL-6, and $\mathrm{IL}-1 \mathrm{~B}$ ) in BALF increased dramatically in the LPS group compared to the sham group; however the level decreased by TXL treatment $(P<0.01$, Figure $4 A)$.

Consistent results were also observed with ELISA, except that $2.5-\mathrm{mg} / \mathrm{kg}$ TXL did not reduce IL-6 levels significantly ( $P<0.01$, Figure $4 B$ ). Collectively, these findings indicated that TXL significantly inhibited sepsis-induced inflammatory response in mice.

\section{$T X L$ regulated Th17/Treg-related cytokine balance in a sepsis model of mice lung tissues}

Flow cytometry analysis was employed to verify the balance between Th17 and Treg cells. It was found that LPSinduced sepsis dramatically increased the proportion of Th17 cells, while TXL effectively eliminated this effect in a dose-dependent manner. For Treg cells, little difference was observed between the LPS group and the sham group whereas TXL treatment remarkably increased the content of Treg cells $(P<0.01$, Figure $5 A)$. Moreover, further investigations proved that LPS increased pro-inflammatory cytokines (IL-17) as well as anti-inflammatory cytokines (IL-10). Nevertheless, TXL treatment dramatically reduced the production of IL-17 and increased the secretion of IL-10 in BALF $(P<0.01$, Figure 5B). Additionally, expression of RORyt, the transcription factor associated with Th17, and Foxp3, the transcription factor associated with Tregs, was up-regulated in the LPS group compared to normal mice; however, TXL notably inhibited RORyt expression and further increased Foxp3 expression in a dose-dependent manner $(P<0.01$, Figure $5 C)$. Taken together, all these investigations illustrated that TXL exerted its protective role in sepsis-induced lung injury by regulating balance between Th17 and Treg cells.

\section{TXL inhibited JAK/STAT3 signaling pathway}

Based on the correlation between TXL and JAK/STAT3 signaling pathway, the expression levels of p-JAK and p-STAT3 were determined to explore the detailed mechanism by which TXL improved Th17/Treg imbalance in sepsis-induced lung damage. The results demonstrated that LPS-induced sepsis increased p-STAT3 and p-JAK levels, which were

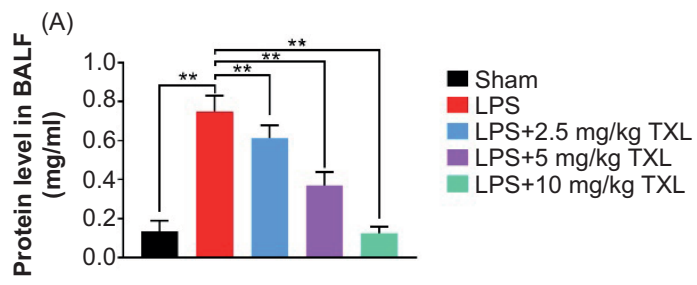

(C)
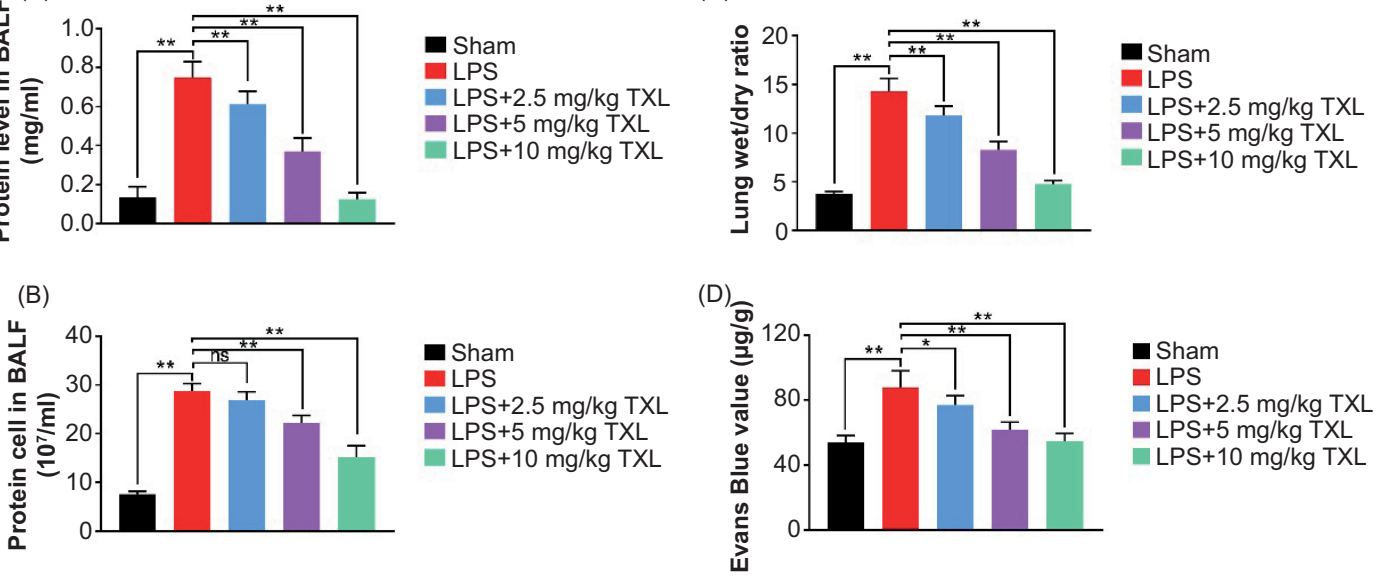

Figure 3 TXL relieved sepsis-induced capillary leakage in the lungs. (A) The protein level in BALF detected by BCA protein assay kit ("*P $<0.01)$. (B) The total cell count in BALF detected by hemacytometry assay ("*P $<0.01)$. (C) Lung wet:dry ratio ("*P<0.01). (D) The Evans blue value detected by Evans blue staining ( ${ }^{* *} \mathrm{P}<0.01$, ${ }^{*} \mathrm{P}<0.05$ ). 
(A)
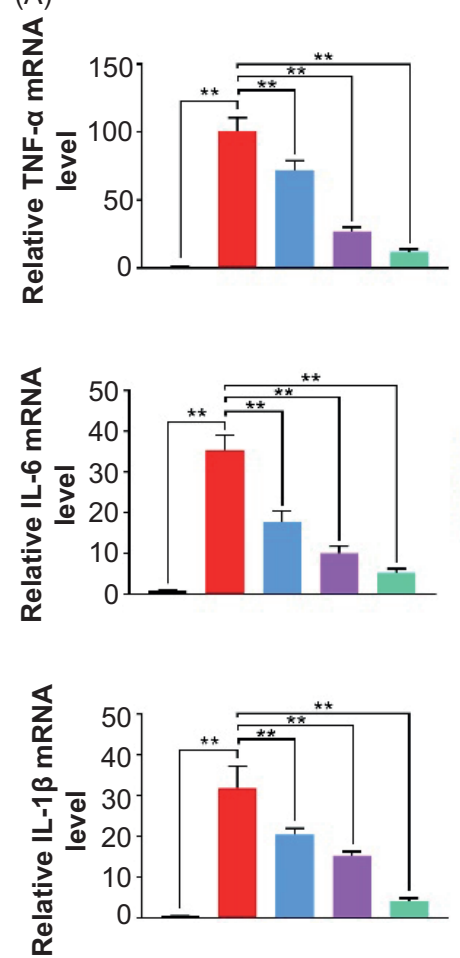

(B)

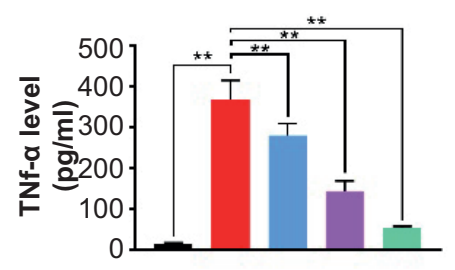

- Sham

- LPS

- LPS $+2.5 \mathrm{mg} / \mathrm{kg}$ TXL

LPS $+5 \mathrm{mg} / \mathrm{kg}$ TXL

- LPS $+10 \mathrm{mg} / \mathrm{kg}$ TXL
- Sham

- LPS

LPS+2.5 mg/kg TXL

- LPS $+5 \mathrm{mg} / \mathrm{kg}$ TXL

LPS+10 $\mathrm{mg} / \mathrm{kg}$ TXL

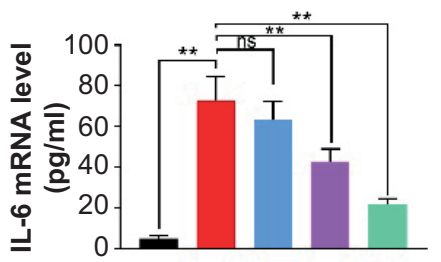

- Sham

- LPS

LPS+2.5 mg/kg TXL

- LPS $+5 \mathrm{mg} / \mathrm{kg}$ TXL

LPS+10 mg/kg TXL

Figure 4 TXL inhibited sepsis-induced inflammatory response in BALF. (A) The mRNA level of pro-inflammatory cytokines detected by qRT-PCR (" $\mathrm{P}<0.01)$. (B) The level of pro-inflammatory cytokines detected by ELISA ("'P $<0.01)$.

efficiently reversed by TXL treatment in a dose-dependent manner $(P<0.01$, Figure 6$)$. These findings revealed that TXL protected mice from sepsis-induced lung injury, at least in part, by suppressing JAK/STAT3 signaling pathway.

\section{Discussion}

Sepsis has been discovered to exhibit complicated and heterogenic characteristics in immune responses, and it obviously becomes difficult to treat patients diagnosed with sepsis. ${ }^{21}$ Therefore, it is essential to find novel agents and elucidate the underlying mechanism for breaking this dilemma. In our present investigation, LPS mice model was employed to reveal that TXL treatment significantly improved survival rate of mice and relieved sepsis-induced lung injury. Moreover, TXL remarkably inhibited sepsis-induced capillary leakage as well as inflammatory response in BALF. Additionally, it was established that TXL regulated Th17/Treg cells balance by modulating JAK/STAT3 signaling pathway. These results proved that TXL protected mice from LPS-caused sepsis by inhibiting inflammatory response.

Being a disease featured with multi-organ failure and even death, sepsis could be aggravated by introducing inflammatory response to LPS. ${ }^{22}$ It has been reported that TXL played a crucial role in anti-inflammation and resisting hepatocellular injury. ${ }^{15}$ However, the detailed role of TXL in LPS-induced sepsis is not revealed so far. Therefore, sepsis mice model induced by LPS was constructed and the role of TXL in LPS-induced sepsis was explored. Consistent with the results of a previous study, ${ }^{23}$ pre-treating with LPS shortened the survival time of mice and injured lung tissues, indicating that the sepsis model was successfully established. However, TXL effectively improved the survival rate of sepsis mice and alleviated lung injury in a dose-dependent manner, which suggested that TXL protected mice from LPS-induced sepsis dramatically. Moreover, vascular leak has been regarded as a significant contributor to promote the development of lung injury, although the underlying mechanism has not been understood. ${ }^{18}$ Findings in the present study established that TXL reduced protein level, lung wet:dry ratio, and total cells in addition to Evans blue value in BALF, confirming that TXL treatment notably alleviated sepsis-induced lung capillary leakage.

It has been reported that inflammatory cytokines increased during LPS-induced sepsis. ${ }^{24,25}$ It was proved that LPS increased the levels of inflammatory cytokines such as TNF- $\alpha$ and IL- 6 , in addition to IL- $B$, which was effectively reversed by $T X L$ treatment. These results illustrated that TXL suppressed pro-inflammatory response remarkably. As two leading $T$ assistant lymphocyte subsets, Th17 and Treg cells are believed to be the vital components maintaining the proper functioning of body's immune system. ${ }^{8}$ Th17 cells originating from CD4+ $T$ cells exert essential effects on inducing graft rejective reaction as well as autoimmune diseases by triggering pro-inflammatory response. ${ }^{26}$ The Th17/Treg balance is tightly associated with body's immune homeostasis. ${ }^{7}$ Moreover, Th17 and Treg cells are indivisible, and the role of Treg cells is closely affected by Th17 cells. ${ }^{27}$ Based on the data, we further explored the relationship between TXL and immune system, especially the Th17/Treg balance. The present study revealed that TXL modulated the balance between Th17 and Treg cells through decreasing LPS-induced increase in Th17 cells and 

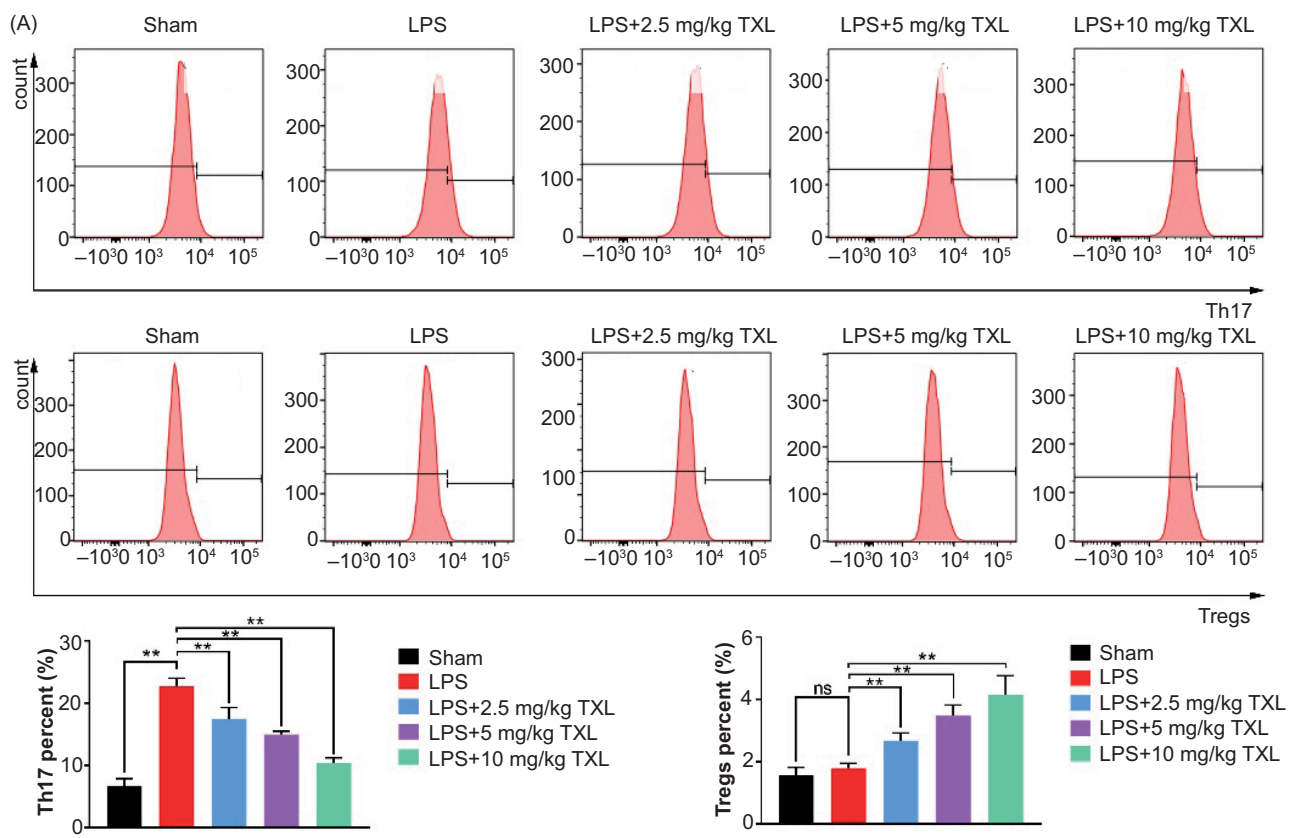

(B)
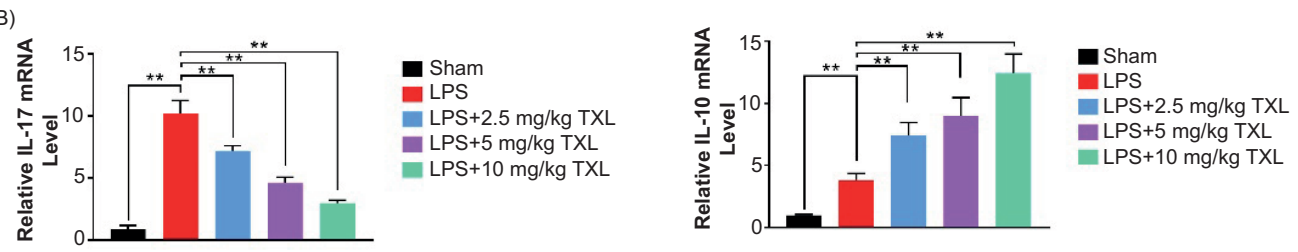

(C)
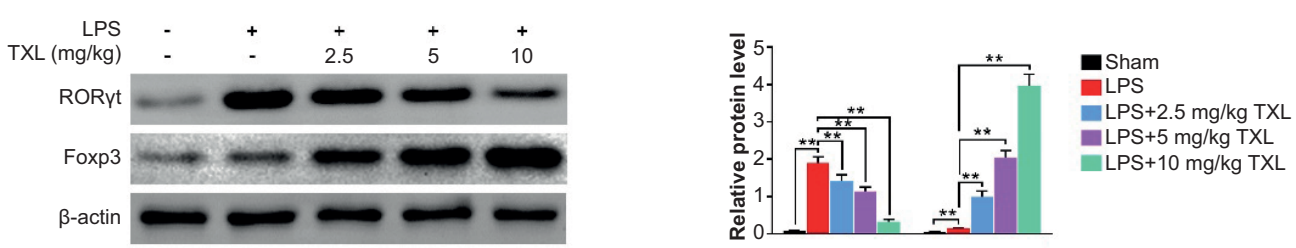

LPS $+2.5 \mathrm{mg} / \mathrm{kg}$ TXL
$\mathrm{LPS}+5 \mathrm{mg} / \mathrm{kg} \mathrm{TXL}$

LPS $+10 \mathrm{mg} / \mathrm{kg}$ TXL

Figure 5 TXL regulated Th17/Treg-related cytokine balance in a sepsis model of mice. (A) The content of Th17 and Treg cells detected by flow cytometry analysis ( $\left.{ }^{* *} \mathrm{P}<0.01\right)$. (B) The mRNA level of inflammatory cytokines detected by $q R T-P C R\left({ }^{* *} P<0.01\right)$. (C) The protein level of relevant transcription factor detected by Western blot assay (" $\mathrm{P}<0.01)$.

increasing LPS-induced decrease in Treg cells. Additionally, Th17 cells have been found to promote inflammatory response by producing pro-inflammatory cytokines such as IL-17, and Treg cells play an immunosuppressive role in various modes such as secreting anti-inflammatory cytokines. ${ }^{17}$ As expected, TXL down-regulated the expressions of IL17 and Th17-related transcription factor but up-regulated the expressions IL-10 and Treg-related transcription factor significantly. To the best of our knowledge, these findings have demonstrated for the first time that $T X L$ regulated the balance between Th17 and Treg cells and the associated inflammatory response.

Provided that TXL exerted its roles in Alzheimer's disease by regulating JAK/STAT3 signaling pathway partly ${ }^{17}$ and signal transducer and activator of transcription (STAT) proteins were crucial for regulating inflammation ${ }^{28}$ and the function of Th17 and Treg cells, ${ }^{29-31}$ we wonder how TXL affected JAK/STAT3 signaling pathway. The results affirmed that TXL treatment inhibited the activation of this pathway in a dose-dependent manner, suggesting that TXL played its protective role in lung capillary leak and lung injury at least partly by inhibiting JAK/STAT3 pathway.

The future studies must investigate how TXL affected JAK/STAT3 signaling pathway, as well as the direct target gene of TXL, which were not investigated in the present study. Moreover, sufficient explorations are required to verify the clinical functions of TXL in subsequent research.

\section{Conclusion}

This investigation elucidated that TXL is capable of inhibiting sepsis-induced capillary leakage and inflammatory response in lung injury, thereby improving the degree of lung damage. Furthermore, TXL plays its protective role by modulating Th17/Treg cells balance via regulating JAK/ STAT 3 signaling pathway. Our present findings provide a promising clinical therapy for treating patients with sepsis. 

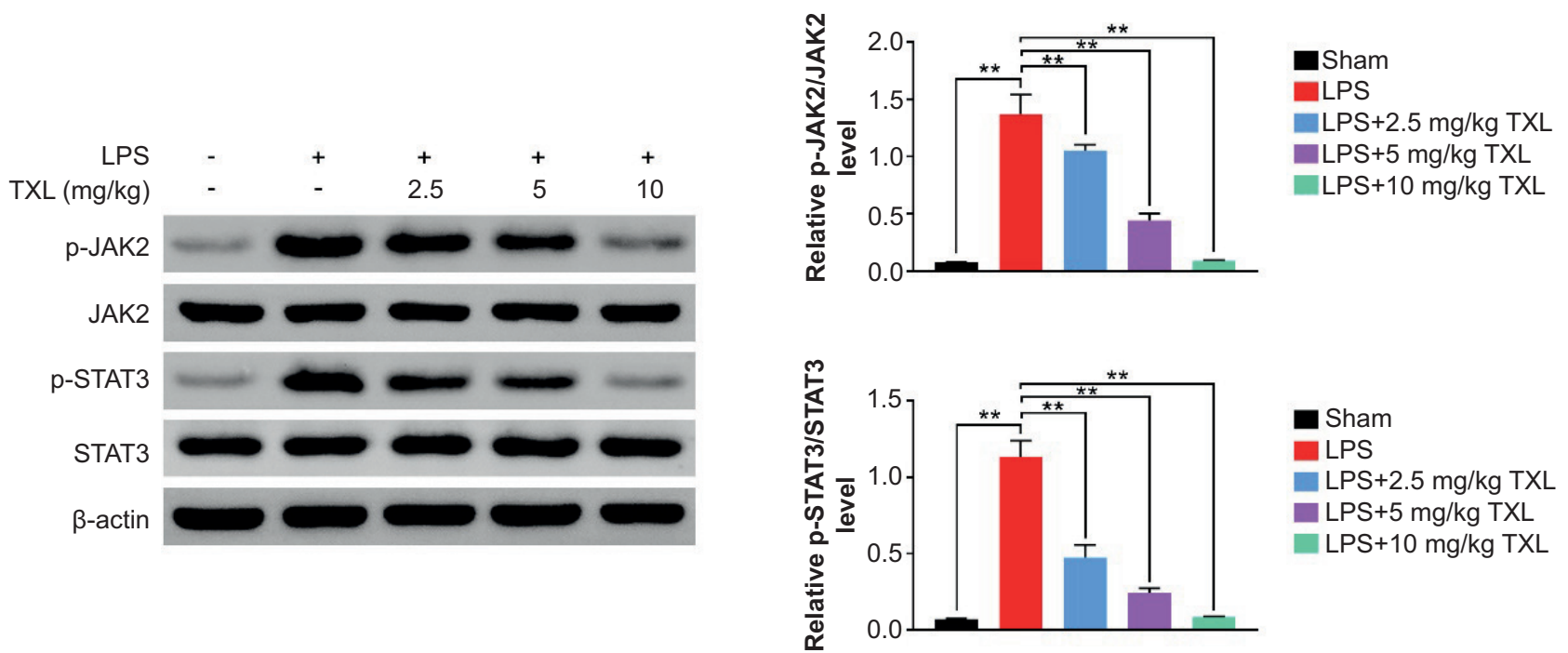

Figure 6 TXL inhibited JAK/STAT3 signaling pathway. The protein level of relevant proteins related to JAK/STAT3 signaling pathway $\left({ }^{* *} \mathrm{P}<0.01\right)$.

\section{Funding}

This work was supported by the Three-year action plan of Shanghai for further accelerating the inheritance, innovation, and development of traditional Chinese medicine (2021-2023) (ZY(2021-2023)-0102), and the Yueyang Hospital Scientific Research Fund Youth Incubation Project (2019YYQ07).

\section{Availability of Data and Materials}

All data generated and analyzed in this study are included in this published article.

\section{Competing Interests}

The authors state that there were no conflicts of interest to disclose.

\section{Contribution of Authors}

Mengwen Shen and Baibai Lin designed the study and supervised data collection. Fenghua Qian analyzed and interpreted the data. Lei Zhao, Yao Xi, and Yiming Qian reviewed the draft and prepared the manuscript for publication. All authors read and approved the final manuscript.

\section{References}

1. Rudd KE, Johnson SC, Agesa KM, Shackelford KA, Tsoi D, Kievlan DR, et al. Global, regional, and national sepsis incidence and mortality, 1990-2017: Analysis for the Global
Burden of Disease Study. Lancet. 2020;395(10219):200-11. https://doi.org/10.1016/S0140-6736(19)32989-7

2. Singer M, Deutschman CS, Seymour CW, Shankar-Hari M, Annane D, Bauer $M$, et al. The Third International Consensus Definitions for Sepsis and Septic Shock (Sepsis-3). JAMA. 2016;315(8):801-10. https://doi.org/10.1001/jama.2016.0287

3. Neil M Goldenberg BES, Arthur S Slutsky, Warren L Lee. Broken barriers: A new take on sepsis pathogenesis. Sci Transl Med. 2011 Jun;3(88):88ps25. https://doi.org/10.1126/ scitranslmed. 3002011

4. Acheampong A, Vincent JL. A positive fluid balance is an independent prognostic factor in patients with sepsis. Crit Care. 2015;19:251. https://doi.org/10.1186/s13054-015-0970-1

5. Schulte W, Bernhagen J, Bucala R. Cytokines in sepsis: Potent immunoregulators and potential therapeutic targets-An updated view. Mediators Inflamm. 2013;2013:165974. https:// doi.org/10.1155/2013/165974

6. Wu TT, Tai YT, Cherng YG, Chen TG, Lin CJ, Chen TL, et al. GATA-2 transduces LPS-induced il-1beta gene expression in macrophages via a toll-like receptor 4/MD88/MAPK-dependent mechanism. PLoS One. 2013;8(8):e72404. https://doi. org/10.1371/journal.pone.0072404

7. Gupta DL, Bhoi S, Mohan T, Galwnkar S, Rao DN. Coexistence of Th1/Th2 and Th17/Treg imbalances in patients with post-traumatic sepsis. Cytokine. 2016;88:214-21. https://doi. org/10.1016/j.cyto.2016.09.010

8. Li J, Li M, Su L, Wang H, Xiao K, Deng J, et al. Alterations of $T$ helper lymphocyte subpopulations in sepsis, severe sepsis, and septic shock: A prospective observational study. Inflammation. 2015;38(3):995-1002. https://doi.org/10.1007/ s10753-014-0063-3

9. Feng $\mathrm{P}$, Yan $\mathrm{R}$, Dai $\mathrm{X}$, Xie $\mathrm{X}$, Wen $\mathrm{H}$, Yang $\mathrm{S}$. The alteration and clinical significance of Th1/Th2/Th17/Treg cells in patients with multiple myeloma. Inflammation. 2015;38(2):705-9. https://doi.org/10.1007/s10753-014-9980-4

10. Wu HP, Chung K, Lin CY, Jiang BY, Chuang DY, Liu YC. Associations of $T$ helper 1, 2, 17 and regulatory $T$ lymphocytes with mortality in severe sepsis. Inflamm Res. 2013;62(8):75163. https://doi.org/10.1007/s00011-013-0630-3

11. Chen X, Feng Y, Shen X, Pan G, Fan G, Gao X, et al. Anti-sepsis protection of Xuebijing injection is mediated by differential 
regulation of pro- and anti-inflammatory Th17 and T regulatory cells in a murine model of polymicrobial sepsis. J Ethnopharmacol. 2018;211:358-65. https://doi.org/10.1016/j. jep.2017.10.001

12. Guo J, Tao W, Tang D, Zhang J. Th17/regulatory T cell imbalance in sepsis patients with multiple organ dysfunction syndrome: Attenuated by high-volume hemofiltration. Int J Artif Organs. 2017;40(11):607-14. https://doi.org/10.5301/ ijao. 5000625

13. Ni J, Zhao Y, Su J, Liu Z, Fang S, Li L, et al. Toddalolactone protects lipopolysaccharide-induced sepsis and attenuates lipopolysaccharide-induced inflammatory response by modulating HMGB1-NF-kappaB translocation. Front Pharmacol. 2020;11:109. https://doi.org/10.3389/fphar.2020.00109

14. Chiu YJ, Chou SC, Chiu CS, Kao CP, Wu KC, Chen CJ, et al. Hepatoprotective effect of the ethanol extract of polygonum orientale on carbon tetrachloride-induced acute liver injury in mice. J Food Drug Anal. 2018;26(1):369-79. https://doi. org/10.1016/j.jfda.2017.04.007

15. Salama SA, Kabel AM. Taxifolin ameliorates iron overload-induced hepatocellular injury: Modulating PI3K/AKT and p38 MAPK signaling, inflammatory response, and hepatocellular regeneration. Chem Biol Interact. 2020;330:109230. https:// doi.org/10.1016/j.cbi.2020.109230

16. Unver E, Tosun M, Olmez H, Kuzucu M, Cimen FK, Suleyman $Z$. The effect of taxifolin on cisplatin-induced pulmonary damage in rats: A biochemical and histopathological evaluation. Mediators Inflamm. 2019;2019:3740867. https://doi. org $/ 10.1155 / 2019 / 3740867$

17. Xia $\mathrm{H}$, Wang $\mathrm{F}$, Wang $M$, Wang J, Sun $\mathrm{S}$, Chen $M$, et al. Maresin1 ameliorates acute lung injury induced by sepsis through regulating Th17/Treg balance. Life Sci. 2020;254:117773. https:// doi.org/10.1016/j.lfs.2020.117773

18. Parikh SM, Mammoto T, Schultz A, Yuan HT, Christiani D, Karumanchi SA, et al. Excess circulating angiopoietin-2 may contribute to pulmonary vascular leak in sepsis in humans. PLoS Med. 2006;3(3):e46. https://doi.org/10.1371/journal. pmed.0030046

19. Thimmulappa RK, Lee H, Rangasamy T, Reddy SP, Yamamoto $\mathrm{M}$, Kensler TW, et al. Nrf2 is a critical regulator of the innate immune response and survival during experimental sepsis. J Clin Invest. 2006;116(4):984-95. https://doi.org/10.1172/ JCI25790

20. Zhang CC, Niu F. LncRNA NEAT1 promotes inflammatory response in sepsis-induced liver injury via the Let-7a/TLR4 axis. Int Immunopharmacol. 2019;75:105731. https://doi. org/10.1016/j.intimp.2019.105731

21. Hwang JS, Kim KH, Park J, Kim SM, Cho H, Lee $Y$, et al. Glucosamine improves survival in a mouse model of sepsis and attenuates sepsis-induced lung injury and inflammation.
J Biol Chem. 2019;294(2):608-22. https://doi.org/10.1074/jbc. RA118.004638

22. Fang J, Qiao F, Tu J, Xu J, Ding F, Liu Y, et al. High expression of long non-coding RNA NEAT1 indicates poor prognosis of human cancer. Oncotarget. 2017;8(28):45918-45927. https:// doi.org/10.18632/oncotarget.17439

23. Han J, Ding R, Zhao D, Zhang Z, Ma X. Unfractionated heparin attenuates lung vascular leak in a mouse model of sepsis: role of RhoA/Rho kinase pathway. Thromb Res. 2013;132(1):e42-7. https://doi.org/10.1016/j.thromres.2013.03.010

24. Thomas RC, Bath MF, Stover CM, Lambert DG, Thompson JP. Exploring LPS-induced sepsis in rats and mice as a model to study potential protective effects of the nociceptin/orphanin FQ system. Peptides. 2014;61:56-60. https://doi.org/10.1016/j. peptides.2014.08.009

25. Hung YL, Fang SH, Wang SC, Cheng WC, Liu PL, Su CC, et al. Corylin protects LPS-induced sepsis and attenuates LPSinduced inflammatory response. Sci Rep. 2017;7:46299. https://doi.org/10.1038/srep46299

26. Afzali B, Lombardi G, Lechler RI, Lord GM. The role of $\mathrm{T}$ helper 17 (Th17) and regulatory $\mathrm{T}$ cells (Treg) in human organ transplantation and autoimmune disease. Clin Exp Immunol. 2007;148(1):32-46. https://doi. org/10.1111/j.1365-2249.2007.03356.x

27. Chen Z, Lin F, Gao Y, Li Z, Zhang J, Xing Y, et al. FOXP3 and RORgammat: transcriptional regulation of Treg and Th17. Int Immunopharmacol. 2011;11(5):536-42. https://doi. org/10.1016/j.intimp.2010.11.008

28. Cardoso CR, Provinciatto PR, Godoi DF, Fonseca MT, Ferreira BR, Teixeira G, et al. The signal transducer and activator of transcription 6 (STAT-6) mediates Th2 inflammation and tissue damage in a murine model of peanut-induced food allergy. Allergol Immunopathol (Madr). 2019;47(6):535-43. https://doi.org/10.1016/j.aller.2019.02.006

29. Zhou J, Li X, Wu X, Zhang T, Zhu Q, Wang X, et al. Exosomes released from tumor-associated macrophages transfer miRNAs that induce a Treg/Th17 cell imbalance in epithelial ovarian cancer. Cancer Immunol Res. 2018;6(12):1578-92. https://doi. org/10.1158/2326-6066.CIR-17-0479

30. Li C, Zhang J, Wang W, Wang H, Zhang Y, Zhang Z. Arsenic trioxide improves Treg and Th17 balance by modulating STAT3 in treatment-naive rheumatoid arthritis patients. Int Immunopharmacol. 2019;73:539-51. https://doi.org/10.1016/j. intimp.2019.05.001

31. Zhao P, Li J, Tian Y, Mao J, Liu X, Feng S, et al. Restoring Th17/ Treg balance via modulation of STAT3 and STAT5 activation contributes to the amelioration of chronic obstructive pulmonary disease by Bufei Yishen formula. J Ethnopharmacol. 2018;217:152-62. https://doi.org/10.1016/j.jep.2018.02.023 